\title{
Legionellosis: Why should I test and report?
}

\author{
Thomas J. Marrie MD, Jason R. Garay BSc BASc MHSc, Erica Weir MD MSc
}

Previously published at www.cmaj.ca

$\mathrm{L}$ egionellosis is a relatively common cause of community-acquired and nosocomial pneumonia (Figure 1) and is identified in about $3 \%-5 \%$ of individuals who are admitted to hospital because of pneumonia in Canada. Guidelines for the empirical treatment of these illnesses call for the use of either fluoroquinolones or macrolides. ${ }^{2}$ Patients and physicians may feel that it is therefore not necessary to confirm the diagnosis of legionellosis in order to treat it. However, from the perspective of public health, it is important that the diagnosis be confirmed and reported so that the possibility of an outbreak and the source of exposure can be investigated. The intent of this primer is to review the organism, clinical manifestations, epidemiology, diagnosis and treatment of legionellosis. We also outline opportunities for public health interventions so that physicians may be more inclined to test and report legionellosis to their local public health units.

\section{What is special about legionellae?}

Legionellae are gram-negative facultative intracellular coccobacilli that measure $0.3-0.9$ microns in width and 2-20 microns in length. They are usually not detectable by Gram staining and require special media for growth in the laboratory. More than 50 species of legionellae, 16 different serogroups of Legionellae pneumophila and 73 serogroups overall have been identified. ${ }^{3}$ Most patients tested for legionellosis have infections of $L$. pneumophila serogroup 1.

Legionella spp. are found in natural and man-made water systems. They are hardy organisms and can survive in most environments for long periods of time. They can withstand temperatures of $0-68^{\circ} \mathrm{C}$ and a $\mathrm{pH}$ range of $5-8.5$. Amoeba and other unicellular organisms in biofilms, which are aggregates of adherent microorganisms attached to a surface and embedded in a self-produced matrix of proteins and polysaccharides (e.g., slime), play a role in the proliferation, development and dissemination of potentially pathogenic Legionella species. ${ }^{4}$ In water distribution systems, they are found in biofilms and are often resistant to biocides and chlorine.

\section{What is the pattern of outbreaks?}

Legionnaires' disease can be acquired by the inhalation of contaminated water aerosols or the aspiration of contaminated

\section{Key points}

- Legionellosis should be reported to public health.

- Under-reporting results in missed sources of exposure.

- All patients with severe pneumonia should be tested for legionellosis.

- Investigation of clusters results in identification of the likely source.

potable water. ${ }^{5}$ Outbreaks have been associated with exposure to various aerosol-producing devices, including showers, ${ }^{6}$ mist machines in grocery stores, ${ }^{7}$ cooling towers, ${ }^{8}$ whirlpool spas, industrial plants ${ }^{9}$ and evapourative condensers. ${ }^{10}$ Legionnaires disease can be acquired at a distance from contaminated sources such as cooling towers or air scrubbers, where the exhaust can be carried by prevailing winds. Cases have been documented as far as $10 \mathrm{~km}$ from such sources. ${ }^{11}$

Some regions of Canada report more cases of legionnaires' disease than others. In a study of community-acquired pneumonia requiring admission to hospital at 15 centres throughout Canada, 3.2\% of the 850 patients had legionnaires disease. ${ }^{1}$ The number of reported cases of legionellosis in the United States increased by $70 \%$ from 1310 cases in 2002 to 2223 cases in 2003. ${ }^{12}$ The eastern US showed the greatest increase in age-adjusted incidence after 2002. Investigators from the Centers for Disease Control and Prevention noted an association between increased rainfall in the mid-Atlantic states and the increasing rate of legionnaires disease. ${ }^{13} \mathrm{~A} 1-\mathrm{cm}$ increase in rainfall was associated with a $2.6 \%$ increase in legionnaires disease. ${ }^{13}$

\section{How does legionellosis present?}

The incubation period of legionnaires' disease is between 2 and 10 days. ${ }^{3}$ Patients with legionnaires' disease are more likely than those whose community-acquired pneumonia was caused by another agent to have myalgia, headache and diar-

From the Faculty of Medicine (Marrie), Dalhousie University, Halifax, NS; Infectious Diseases Control Division (Garay), Public Health Branch York Region Community and Health Services, Newmarket, Ont.; and Community Health and Epidemiology (Weir), Queen's University, Kingston, Ont.

CMAJ 2010. DOI:10.1503/cmaj.082030 
rhea; they also have a higher mean oral temperature at the time of presentation. ${ }^{1}$ Patients with legionnaires disease typically present to hospital sooner after the onset of symptoms. Legionella has features that are distinct from bacteremic pneumococcal pneumonia (Table 1). ${ }^{14}$ Features not usually associated with legionellosis included pleuritic chest pain, previous upper respiratory tract infection and purulent sputum..$^{14}$ There are no individual clinical features that allow physicians to make a diagnosis of legionnaires' disease; however, all patients with severe pneumonia should be tested for legionnaires' disease. Older people ( $\geq 65 \mathrm{yr}$ ) with legionnaires' disease often have more comorbid illnesses than younger people, yet older people present less frequently with fever, nonrespiratory symptoms and laboratory abnormalities. This might be explained by the intracellular nature of the pathogen and altered cell-mediated immunity in elderly hosts.

In a study comparing the radiographic features of legionnaires' disease, pneumococcal pneumonia, mycoplasma pneumonia and psittacosis, Macfarlane and colleagues ${ }^{15}$ noted that progression of pneumonia following hospital admission was a particular feature of legionnaires' disease, occurring in $65 \%(30 / 46)$ of patients, compared with $51 \%$ (14/27) of patients with bacteremic pneumococcal pneumonia. Residual intrapulmonary streaky opacities remained in over a quarter of the survivors of legionnaires' disease. ${ }^{15}$ About half of the patients with legionnaires' disease have unilateral pneumonic involvement throughout the course of their illness. The lower lobes are most commonly involved (Figure 2), and pleural effusions are seen in about 35\% of patients. Additional laboratory findings that can accompany legionellosis are shown in Box $1 .{ }^{16}$

Legionellosis may also occur as a nonpneumonic form,

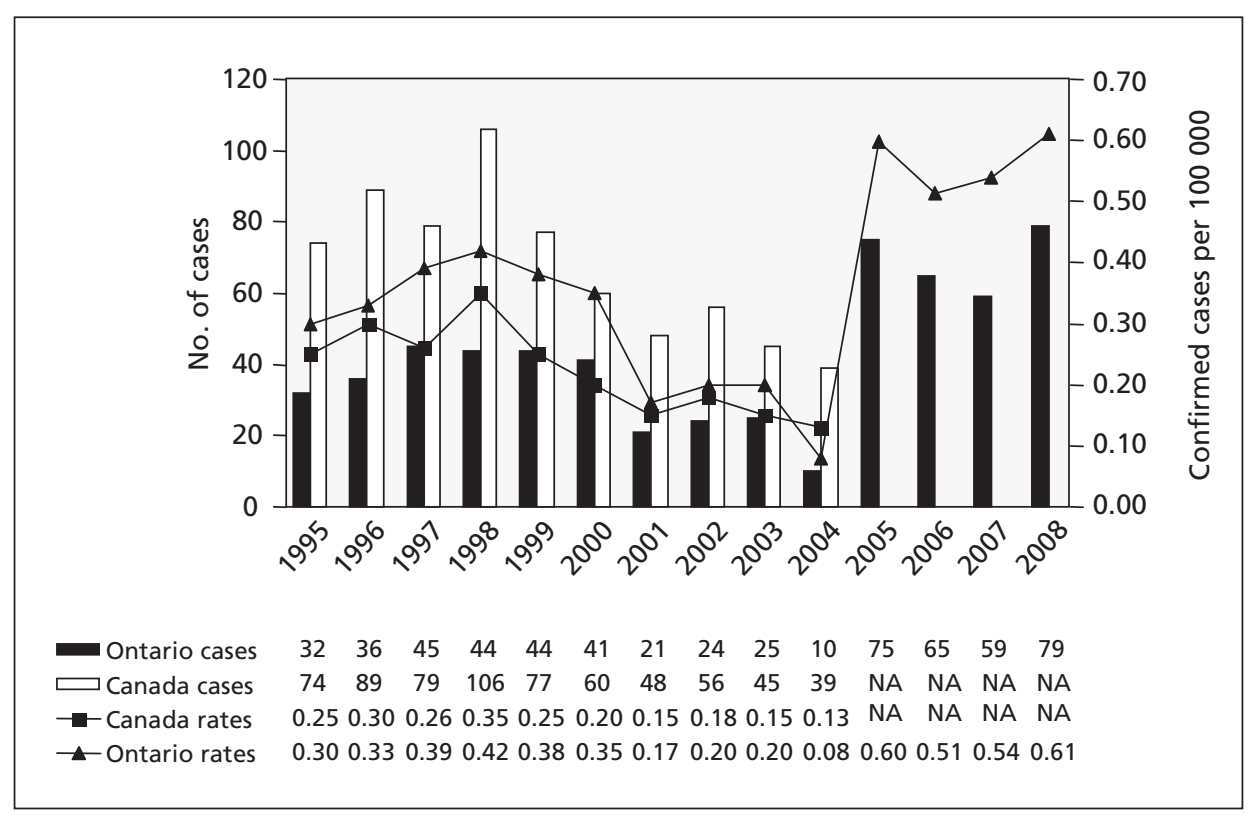

Figure 1: Number and rate of legionellosis cases per 100000 population in Ontario and Canada. Sources: Public Health Agency of Canada Notifiable Diseases Online (extracted June $11,2010)$ and the Ontario Ministry of Health and Long-Term Care, Public Health Ontario Portal (extracted June 11, 2010). NA = Data not available as of June 11, 2010.
Table 1: Features associated with Legionella compared with bacteremic pneumococcal pneumonia ${ }^{14}$

\begin{tabular}{lc}
\hline Feature & Odds ratio $(95 \% \mathrm{Cl})$ \\
\hline Male sex & $4.5(1.48-14.5)$ \\
Heavy alcohol use & $4.8(1.39-16.42)$ \\
Previous $\beta$-lactam therapy & $19.9(3.47-114.2)$ \\
\hline Axillary temperature $>39$ & $10.3(2.71-38.84)$ \\
Myalgia & $8.5(2.35-30.74)$ \\
Gastrointestinal symptoms & $3.5(1.01-12.81)$ \\
\hline
\end{tabular}

Note: $\mathrm{Cl}=$ confidence interval.

such as Pontiac fever. In July 1968, an illness consisting of fever, headache, myalgia and malaise with a very high attack rate $(95 \%)$ occurred among employees in a new county health department building in Pontiac, Michigan. Other symptoms included dizziness, painful stiff neck, nausea, chest pain, joint pain, sore throat, sore eyes, abdominal pain, confusion, coryza, photophobia, diarrhea, poor coordination, anorexia, nose bleed, vomiting, insomnia, bizarre dreams, rash and irritability. The acute illness lasted two to five days, although many experienced weakness and fatigue afterwards. ${ }^{17}$

The link to Legionella species is typically made by culturing legionella from water samples of likely sources and by conducting serologic and/or urine antigen testing in cases. ${ }^{17}$

\section{What tests can confirm the diagnosis?}

The Infectious Diseases Society of America and the American Thoracic Society have developed consensus guidelines for the management of community-acquired pneumonia in adults. ${ }^{18}$ Almost all of the major decisions regarding management, including diagnostic and treatment issues, revolve around the initial assessment of severity. According to these guidelines, patients with severe community-acquired pneumonia should, at a minimum, have blood samples drawn for culture, urinary antigen tests for L. pneumophila and Streptococcus pneumonia and expectorated sputum samples collected for culture.

The clinical diagnosis of legionnaires' disease can be confirmed by the isolation of the organism from sputum, bronchoalveolar lavage fluid, pleural fluid or pulmonary tissue. Detection of the antigen in the urine is a useful test for $L$. pneumophila serogroup 1 . Respiratory specimens can also be tested for the presence of the organism using a 
direct fluorescent antibody technique or by polymerase chain reaction, although these are not available in many settings. Detection of the legionella antigen in urine is quick, usually with a turn around time of about one hour. In a recent meta-analysis, the pooled sensitivity of using the urine antigen test to detect legionella was 0.74 (95\% confidence interval $[\mathrm{CI}] 0.68-0.81$ ) and specificity was 0.99 (95\% CI 0.984-0.997). ${ }^{19}$ The rate of a positive antigen test result depends on the severity of the disease, with a positive result occurring in $40 \%-53 \%$ of mild cases and $88 \%-100 \%$ of severe cases. ${ }^{20}$ The antigen can persist in urine for weeks to months.

Antibodies to Legionella spp. can be detected in acute and convalescent serum samples by several techniques. The convalescent sample should be collected no sooner than six weeks after the acute phase. In some documented cases of legionnaires disease, seroconversion had not occurred by 12 weeks. Recent studies have shown that a previously accepted diagnostic criterion of a single antibody titer of $\geq 1: 256$ is not valid. ${ }^{21} \mathrm{~A}$ four-fold rise in antibody titer between the acute and convalescent phase samples is good evidence that infection with Legionella spp. has occurred.

Data from a prospective, nonrandomized study indicate that levofloxacin (and presumably other quinolones) is superior to macrolides for the treatment of severe legionnaires' disease. ${ }^{22}$ In this study, which was performed in Murcia, Spain, $3.4 \%$ of patients who received levofloxacin had complications, compared with $27.2 \%$ of those who received macrolides. The patients who received levofloxacin also had a shorter hospital stay (5.5 d v. $11.3 \mathrm{~d})$. The addition of

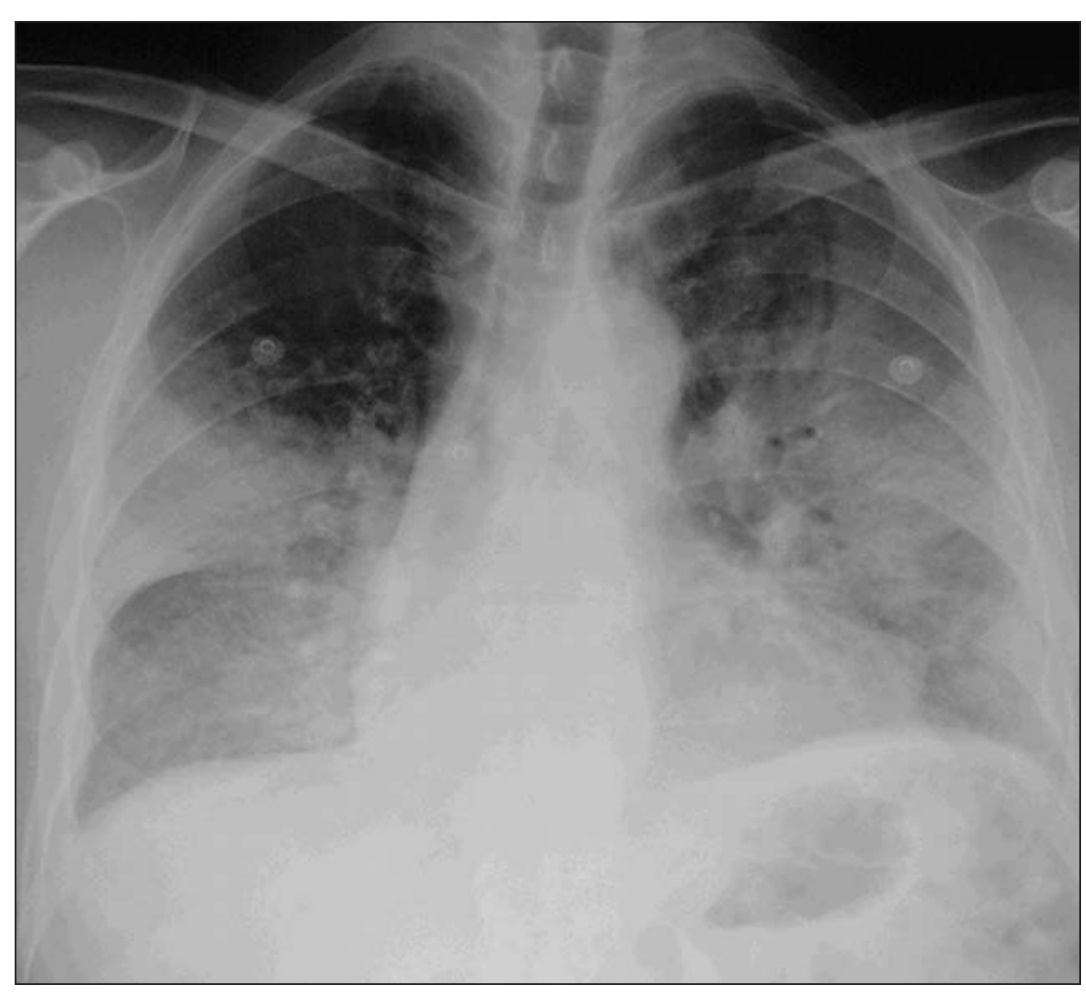

Figure 2: Chest radiograph in a confirmed case of legionellosis with multilobar opacifications.

\section{Box 1: Laboratory findings that may accompany} legionellosis ${ }^{16}$

- Microscopic hematuria

- Hyponatremia

- Elevated liver enzymes

- Hypophosphatemia

- Elevated creatinine phosphokinase

rifampin provided no additional benefit over levofloxacin alone. Azithromycin appears to be the best macrolide for the treatment of legionnaires disease.

\section{Why should it be reported?}

Legionellosis has been a nationally notifiable disease in Canada since 1986. Although reporting by health care providers and laboratories is legislated, underreporting of the disease is common because sporadic cases are often treated without the use of empirical tests to confirm the diagnosis. ${ }^{23}$ Individual cases of legionellosis should be fully investigated in a timely manner because they may indicate a larger pending outbreak.

Risk settings for legionellosis exposure can include hot water systems, air cooling towers, spas or other publicly accessible sites; individual cases are often related to broader clusters with common exposure characteristics. ${ }^{24}$ Geographic clusters of legionellosis have frequently been associated with water cooling towers and air conditioning systems that facilitate the transport of water molecules containing Legionella spp. into the surrounding air supply. ${ }^{25}$ Cluster investigations have often identified proximity to these types of facilities as the most likely source of exposure. Conversely, institution-based Legionella clusters are typically associated with facility-based sources of contamination, including hot and cold water systems. Environmental swabbing within these types of institutions have yielded positive Legionella cultures in shower heads, treatment or recreational water sources, respiratory therapy devices and ice machines. ${ }^{24}$

Investigations of potential clusters of cases of legionellosis often involve mapping the locations (Figure 3 ) and the movement of patients over a specified period of time, along with mapping the location of possible sources of exposure. Using this technique, patterns of shared exposures between cases become evident. ${ }^{24}$ These sources can then be sampled and tested for the presence of Legionella species. Plume modeling, which maps airborne particle dispersion under specified meterological conditions, can also aid in identifying possible sources, exposures and transmissions.

Control of Legionella, whether environmentally or within institutions, is hampered 


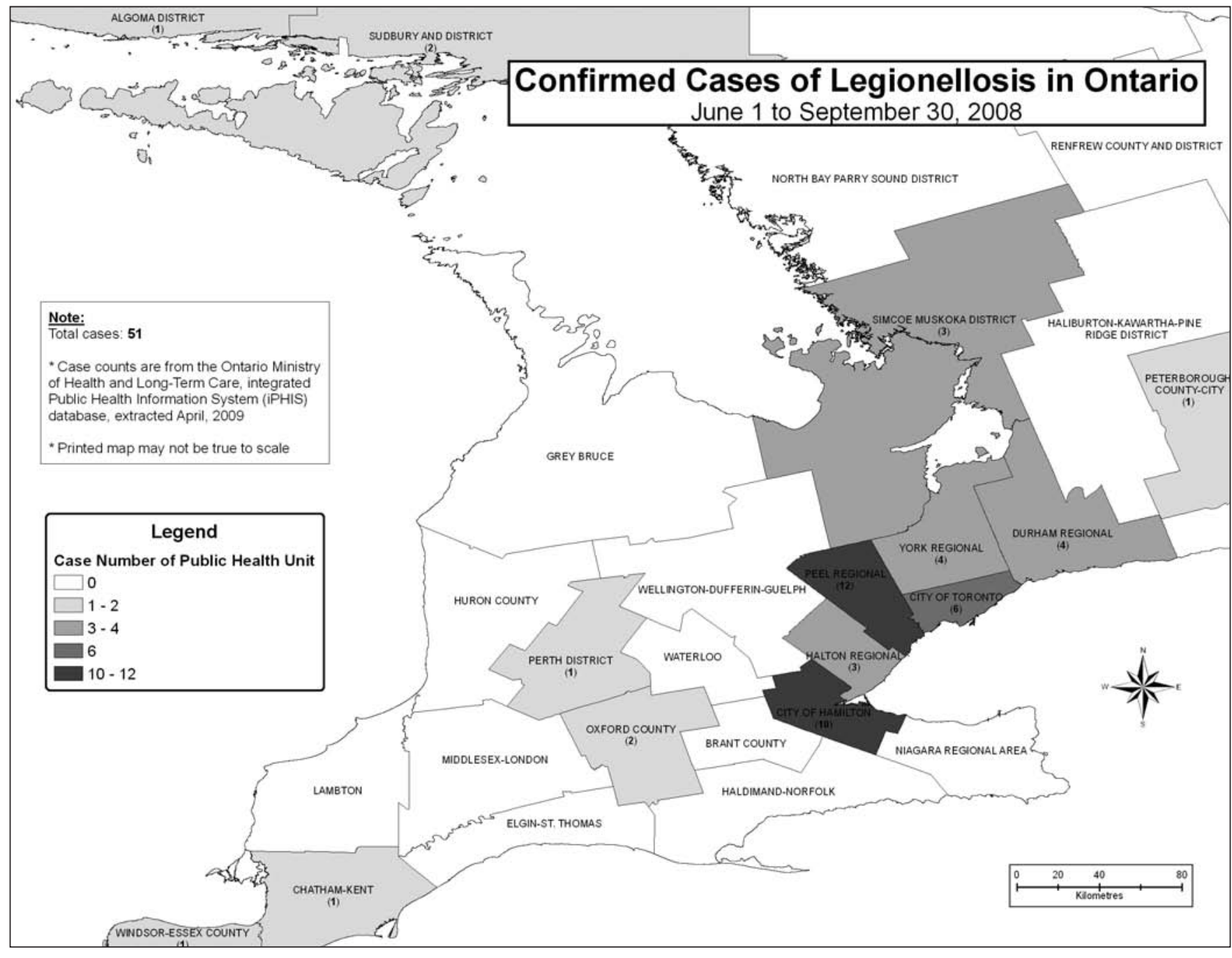

Figure 3: Confirmed cases of legionellosis in Ontario from June 1 to Sept. 30, 2008. Reproduced with permission from the Ministry of Health and Long-Term Care.

by the ubiquitous nature of the organism. Outbreak response to Legionella infection often involves the identification and remediation of suspect exposure sites to eliminate the source of the pathogen. Control methods include superheating, hyperchlorination, source-based ultraviolet treatment, physical cleaning of outlet points and microbial filtration. ${ }^{24}$ However, it is generally agreed that Legionella

\section{Box 2: Data that physicians should provide to public} health about confirmed cases of legionellosis

- Patient's first and last name

- Episode date (defined as either onset of symptoms, specimen collection date, laboratory report date)

- Patient's birth date

- Patient's address and telephone number

- Date and method of diagnosis

- Patient's symptoms

- Tests performed and results cannot be completely eradicated from water systems. Indeed, long-term control of the organism, and subsequent prevention of disease, can only be achieved through regular treatment and management of water systems following strict guidelines for the prevention of microbial growth.

Robust local surveillance systems supplied with timely clinical case data will assist in the early identification of clusters and risk settings and the early application of remediation measures to limit further transmission of the disease. This is why health care providers should test for legionellosis when it is suspected and report cases (Box 2) to the local public health authorities.

This article has been peer reviewed.

Competing interests: None declared for Jason Garay and Erica Weir. Tom Marrie is a board member of Capital District Health Authority and an advisory board member of Wyeth. He has received speaker fees and grants from Wyeth for pneumococcal vaccines and is Dean of the Faculty of Medicine at Dalhousie University.

Contributors: Each author made a substantial contribution to the conception and acquisition of data, drafting and final approval of the manuscript. 


\section{REFERENCES}

1. Marrie TJ, de Carolis E, Yu VL et al.; Canadian community-acquired pneumonia investigators. Legionnaires' Disease - results of a multicentre Canadian study. Can J Infect Dis 2003;14:154-8.

2. Institute for Clinical Systems Improvement. Health care guideline: communityacquired pneumonia in adults. Bloomington (MN): Institute for Clinical Systems Improvement; 2006. Available: www.icsi.org/pneumonia_community-acquired /community-acquired pneumonia_2.html (accessed 2009 Mar. 23).

3. Luck PC. Diagnostics and clinical disease treatment. In: Heuner K, Swanson M, editors. Legionella - molecular microbiology. Norfolk (UK): Caister Academic Press; 2008. p. 19-34.

4. Lau h, Ashbolt NJ. The role of biofilms and protozoa in legionella pathogenesis implications for drinking water. J Appl Microbiol 2009;107:368-78.

5. Hanrahan JP, Morse DL, Scharf VB, et al. A community hospital outbreak of Legionellosis: transmission by potable hot water. Am J Epidemiol 1987;125:639-49.

6. Delia S, Lagana P, Minutoli E. Occurrence of legionella in beach shower facilities. J Prev Med Hyg 2007;48:114-7.

7. Mahoney FJ, Hoge CW, Farley TA, et al. Community-wide outbreak of Legionnaires' disease associated with a grocery store mist machine. J Infect Dis 1992; 165:736-9.

8. Dondero TJ Jr, Rendtorff RC, Mallison GF, et al. An outbreak of Legionnaires disease associated with a contaminated air-conditioning cooling tower. $N$ Engl J Med 1980;302:365-70.

9. Borgen K, Aaberge I, Werner-Johansen O, et al. A cluster of Legionnaire disease linked to an Industrial plant in southeast Norway, June-July 2008. Euro Surveill 2008;13:pii:18985.

10. Cordes LG, Fraser DW, Skaliy P, et al. Legionnaires' disease outbreak at an Atlanta, Georgia, country club: Evidence for spread from an evaporative condenser. Am J Epidemiol 1980;111:425-31.

11. Nygard K, Werner-Johansen O, Ronsen S, et al. An outbreak of Legionnaires' disease caused by long distance spread from an industrial air scrubber in Sarpsborg, Norway. Clin Infect Dis 2008;46:61-9.

12. Neil K, Berkelman R. Increasing incidence of Legionellosis in the United States, 1990-2005. Clin Infect Dis 2008;47:591-4.

13. Hicks LA, Rose CE Jr, Fields BS, et al. Increased rainfall is associated with increased risk for Legionellosis. Epidemiol Infect 2007;135:811-7.

14. Fernandez-Sabe N, Reson B, Carratala J, et al. Clinical diagnosis of Legionella pneumonia revisited: Evaluation of the community-based pneumonia incidence study group scoring system. Clin Infect Dis 2003;37:483-9.

15. Macfarlane JT, Miller AC, Smith WHR, et al. Comparative radiographic features of community acquired legionnaires' disease, pneumococcal pneumonia, mycoplasma pneumonia, and psittacosis. Thorax 1984;39:28-33.

16. Meyer RD. Legionella infections: A review of five years of research. Rev Infect Dis 1983;5:258-78.

17. Modi A, Gardner J, Lighton L, Pontiac fever outbreak associated with a spa-pool, United Kingdom, April 2008. Euro Surveill. 2008;13:pii: 18934.

18. Mandell 1, Wunderink R, Anzueto A, et al. Infectious disease society of America/American thoracic society consensus guidelines on the management of community-acquired pneumonia in adults. Clin Infect Dis 2007;44:S27-72.

19. Shimada T, Noguchi Y, Jackson JL, et al. Systematic review and meta-analysis: urinary tests for Legionellosis. Chest 2009;136:1576-85.

20. Waterer GW, Baselski VS, Wunderink RG. Legionella and community-acquired pneumonia: A review of current diagnostic tests from a clinician's viewpoint. Am J Med 2001;110:41-8.
21. Plouffe JF, File TM Jr, Breiman RF, et al. Reevaluation of the definition of Legionnaires' disease: use of the urinary antigen assay. Community Based Pneumonia Incidence Study Group. Clin Infect Dis 1995;20:1286-91.

22. Blazquez Garrido RM, Parra FJE, Frances LA, et al. Antimicrobial chemotherapy for Legionnaires' disease; Levofloxacin versus macrolides. Clin Infect Dis 2005;40:800-6.

23. Edelstein PH, Ciancioto NP. Legionnella. In: Mandell, Douglas and Bennett's principles and practice of infectious diseases, $6^{\text {th }}$ edition. Philadelphia (PA): Elsevier; 2005. p 2711-24.

24. Guideline on Management of Legionella Incidents. Outbreaks and Clusters in the Community. Health Protection Network Scottish Guidance 2. Glasgow (Scotland): Health Protection Scotland; 2009.

25. Moore Matthew R, Fields Barry S. Legionellosis. In: Maxcy-Rosenau-Last public health and preventive medicine, 15th edition. New York (NY): McGraw Hill Medical; 2008. p. 311-3.

\section{Correspondence to: Dr. Erica Weir, Community Health and}

Epidemiology, Queen's University, Kingston ON;

erica.weir@york.ca

\section{Resources}

- Infectious Diseases Society of America/American Thoracic Society Consensus Guidelines on the Management of Community-Acquired Pneumonia in Adults www.journals.uchicago.edu/doi/pdf/10.1086/511159\%20

- European Guidelines for Control and Prevention of Travel Associated Legionnaires' Disease www.ewgli.org/data/european_guidelines/european guidelines_jan05.pdf

- Centre for Disease Control and prevention legionellosis resource site www.cdc.gov/legionella/references.htm

Primer articles are brief narrative reviews on interesting, important and timely clinical topics. The articles emphasize clinical application and are based on the best evidence available. Author instructions are available at cmaj.ca. Submit Primers (maximum 1800 words and up to 20 references) to http://mc.manuscriptcentral.com/cmaj or email diane.kelsall @ cmaj.ca to discuss ideas.

\section{Atacand}

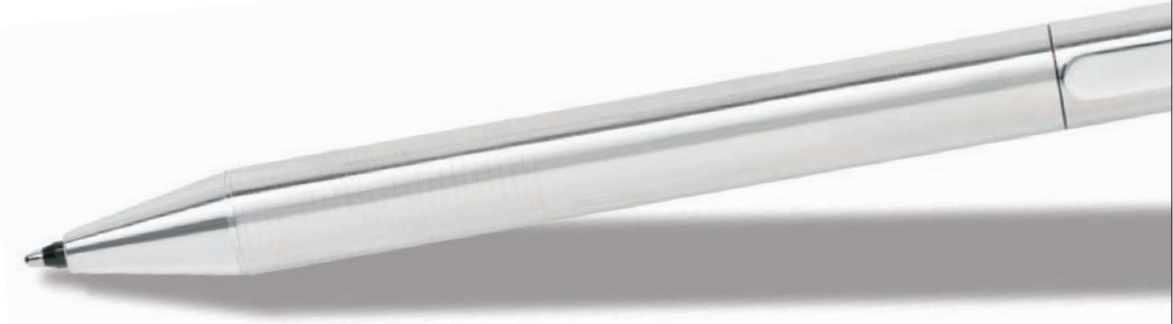

"Atacand"

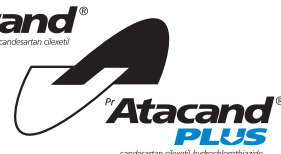

